\title{
CALCULATION OF BEAM-LOADED Q IN HIGH-POWER KLYSTRONS*
}

\author{
J. DeFord and B. Held, Simulation Technology \& Applied Research, Inc., Mequon, WI 53092, \\ U.S.A. \\ K. Ko and V. Ivanov, SLAC, Menlo Park, CA 94025, U.S.A.
}

\begin{abstract}
Instabilities in the gun region of a high-power klystron can occur when there is positive feedback between a mode and an induced current on the quasi-steady state beam emitted by the gun cathode[1]. This instability is dependent on the gun voltage, and is predicted on the basis of a negative total Q. The established method for computing the beam-loaded $\mathrm{Q}$ of a cavity involves using a time-dependent electromagnetic particle-in-cell (PIC) code to track beam particles through the quasi-static gun fields perturbed by the electromagnetic fields of a cavity eigenmode[2]. The energy imparted to the beam by the mode is obtained by integrating the Lorentz force along the particle tracks, and this quantity is simply related to the beam-loaded Q. We have developed an alternative approach that yields comparable accuracy but is computationally much simpler. The new method is based on a time-independent electrostatic PIC calculation, resulting in much faster solutions without loss of accuracy. We will present the theory and implementation of the new method, as well as benchmarks and results from analysis of the XP-4 klystron that show a potential instability near $3 \mathrm{GHz}$.
\end{abstract}

\section{INTRODUCTION}

In designing high power klystrons the primary focus is on power generation and both analytical methods and numerical codes exist for optimizing the tube's efficiency and performance. But often, spurious oscillations at frequencies other than the operating frequency have hampered the progress towards higher power output. These are parasitic modes which, when driven by the beam and not sufficiently loaded down, grow to disrupt the tube's operation. They have been found in the gun region and in the RF circuit but at present there are no reliable tools for predicting which of the many modes is a candidate for causing this type of failure.

Spurious oscillations in the klystron gun and in the circuit are known to grow from noise on the beam, and should therefore be amenable to a small-signal analysis based on the reaction of the particles to a small RF perturbation of the static fields, the perturbation coming from a particular resonant mode of the structure. In the small signal limit the energy coupled to the beam by a mode can be used to compute the beam-loaded $\mathrm{Q}$ for the mode. With additional knowledge of the external $\mathrm{Q}$ for the mode it is possible to compute the total $\mathrm{Q}$, and a negative total Q indicates instability.

In this paper we describe one approach to computing the total Q of modes in the gun and cavities in the circuit. We show comparisons with published analytic and computed results, and also apply the approach to the analysis of the XP-4 klystron under development at SLAC.

\section{COMPUTATION OF PARTICLE EXIT ENERGY}

The approach is based on computing the difference in energy of particles that are tracked alternately through static fields in the structure and then again with the addition of a small RF perturbation based on an eigenmode that is computed separately. Particle tracks are determined using an electrostatic PIC code that includes the effects of self-magnetic fields and space charge. The space charge field and self magnetic field are exported, along with the tracks for representative test particles, for use in the integration process described below.

To determine the exit energy of a particle that traverses a specified path through prescribed static fields perturbed by an RF mode field, its velocity and position along the path are computed by solving the following equation of motion:

$$
\dot{p}_{z}=q E_{z} \rightarrow m_{0} v_{z} \frac{d\left(\mathcal{W}_{z}\right)}{d z}=q_{0} E_{z}
$$

Integrating this expression over a short segment of the particle track (short enough that we can neglect the variation of the electric field both in time and space as the particle traverses the segment) yields the velocity at the end of the segment as a function of velocity at the beginning of the segment and the integral of the axial electric field along the segment:

$$
\beta_{1}^{2}=1-\frac{1-\beta_{0}^{2}}{\left(1-\frac{q_{0} \Delta V}{m_{0} c_{0}^{2}} \sqrt{1-\beta_{0}^{2}}\right)^{2}}
$$

where $\Delta V$ is given by:

$$
\Delta V=\int_{z_{0}}^{z_{0}+\Delta z} E_{z}(z) d z
$$

The electric field can be expanded in a Taylor series about $z_{0}$ to evaluate this integral, and to first order in $\Delta z$ we get:

$$
\Delta V=\int_{z_{0}}^{z_{0}+\Delta z} E_{z}(z) d z \approx E_{z}\left(z_{0}\right) \Delta z\left[1+\frac{\Delta z}{2}\left(\frac{i \omega}{v_{0}}+\frac{f^{\prime}\left(z_{0}\right)}{f\left(z_{0}\right)}\right)\right]
$$

where $f(z)$ is the particle trajectory. After pushing the particle along the segment, we update the time using the average velocity along the segment.

The particle exit velocities are used to compute total beam energy with and without RF fields, so it is important the velocities computed by the method are accurate. It is only the difference between particle velocities/energies in static fields and those perturbed by RF fields that are used 
in the beam-loaded $\mathrm{Q}$ computation, but an inaccurate computation of exit quantities in static fields would indicate fundamental problems with the integration of the force equation.

The computed final particle velocities were compared with the Poisson 2 [3] values for a gun voltage of $100 \mathrm{KV}$, and the maximum difference was less than $0.4 \%$ for all particles, with the largest errors occurring on the particle tracks with the largest curvature near the beam edge. These comparisons tended to improve with increasing gun voltage. The total beam power at the exit plane was also compared, and this showed very good agreement between the perturbation method and the Poisson 2 code, with a maximum error of $0.2 \%$.

\section{COMPARISON TO BRANCH RESULT}

Consider a simple circular pillbox of radius $a$ and axial length $d$, with a zero-radius beam on axis. For the case of purely axial mode fields and sub-relativistic particles, Branch [4] derived the following expression for power flow from the mode to the beam:

$$
\begin{gathered}
P_{b}=-\frac{j I_{0} E_{m}^{2}}{8 V_{0}} \int_{-\infty-\infty}^{\infty} \int^{\infty}\left(z_{2}-z_{1}\right) \beta_{e} g\left(z_{2}\right) g^{*}\left(z_{1}\right) . \\
e^{j \beta_{e}\left(z_{2}-z_{1}\right)} d z_{1} d z_{2}
\end{gathered}
$$

where $E_{m}$ is the maximum instantaneous electric field strength of the mode anywhere in the gap along the particle trajectory, $g(z)$ is the normalized field profile in the gap, $I_{0}$ is the beam current, and

$$
\beta_{e}=\frac{\omega}{u_{0}} \quad V_{0}=\frac{1}{e}\left(\gamma_{0}-1\right) m_{0} c^{2}
$$

with $u_{0}$ and $\gamma_{0}$ corresponding to the particle entrance velocity. When the mode pattern is axially invariant, the power expression evaluates to:

$$
P_{b}=\frac{I_{0} E_{m}^{2}}{4 V_{0} \beta_{e}^{2}}\left[2-2 \cos \left(\beta_{e} d\right)-\beta_{e} d \sin \left(\beta_{e} d\right)\right]
$$

Note that the power transfer is negative for certain values transit angle, meaning that the mode is extracting energy from the beam.

The beam-loaded Q for the mode is given by

$$
Q_{b}=\frac{\omega U_{0}}{P_{b}}
$$

Rewriting Eqn. 7 in terms of the transit angle $\phi=\beta_{e} d$, and substituting into Eqn. 8 yields the following result for the fundamental mode:

$$
\begin{aligned}
& Q_{b}=\frac{\omega U_{0}}{P_{b}}=\frac{U_{0} c_{0} x_{01}}{a P_{b}} \\
& =\frac{2 \pi a x_{01} V_{0} J_{1}^{2}\left(x_{01}\right)}{I_{0} \eta_{0} d}\left[\frac{\phi^{2}}{2-2 \cos (\phi)-\phi \sin (\phi)}\right]
\end{aligned}
$$

A comparison between analytic and computed results for the fundamental mode in a pillbox of dimension $a=10$ $\mathrm{cm}$ and $d=7.5 \mathrm{~cm}$ is shown in Fig. 2 .

\section{KLYSTRON BUNCHING CAVITY}

This benchmark problem establishes the utility of the perturbation method for analyzing the $2 \mathrm{D}$ sections of the circuit, and it involves the computation of beam-loaded Q value for a klystron bunching cavity that was analyzed by Becker, et al [2]. It is assumed that the beam is confined to the axis, and it has an input energy of $116 \mathrm{KV}$ and $88 \mathrm{~A}$ current. The geometry of the cavity is shown in Fig. 3. The fundamental mode in this cavity oscillates at $2747 \mathrm{MHz}$, and Becker's paper lists two results for the beam-loaded Q of this mode based on two different methods of calculation: 87.2 and 88.7. The perturbation method described herein yields 89.8 .

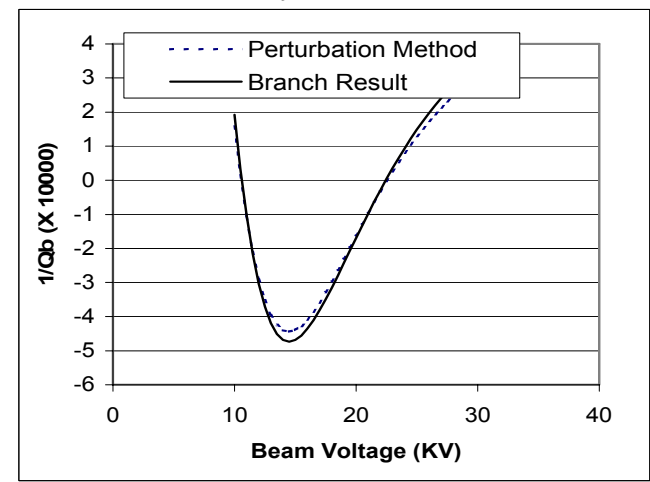

Figure 2: Comparison of computed vs. analytic $\mathrm{Q}_{\mathrm{b}}$ values vs. beam voltage for simple cavity $(\mathrm{d}=7.5 \mathrm{~cm})$.

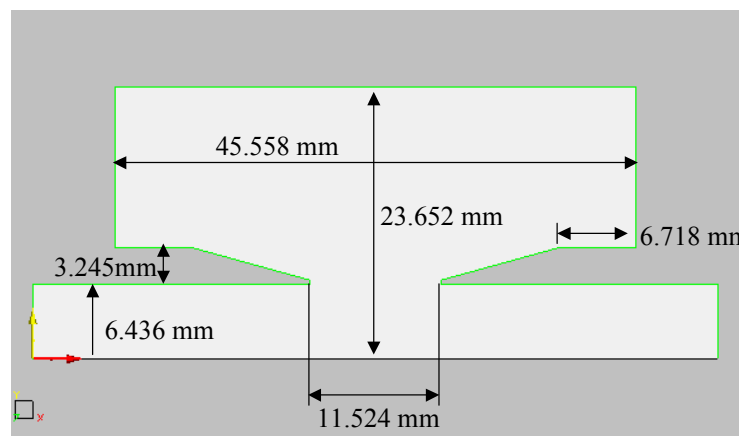

Figure 3: Klystron bunching cavity geometry.

\section{ANALYSIS OF XP-4 GUN REGION}

The geometry of the XP-4 gun is shown in Fig 4, consisting of a vacuum region separated from an oil tank/RF feed by a ceramic window[5]. 


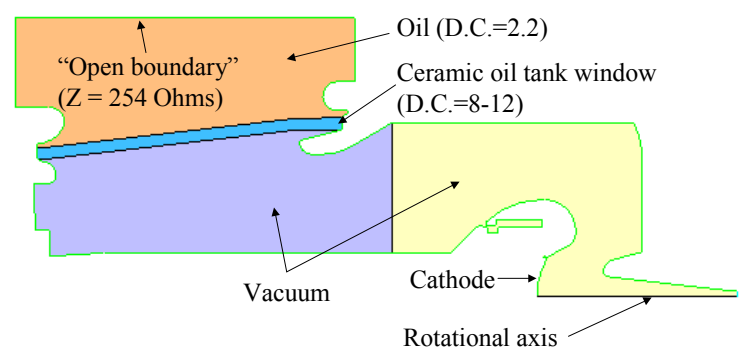

Figure 4: R-Z cross-section of XP-4 klystron gun region. Note that there is no physical divider between the two colors in the vacuum region.

For five different values of the ceramic window dielectric constant $(8,9,10,11$, and 12) all monopole eigenmodes were extracted for this structure between $100 \mathrm{MHz}$ and $5 \mathrm{GHz}$ using the OM2P eigensolver. The total "cold" Q of each mode was also computed by the eigensolver. The modes were extracted in groups of 30 , and approximately 215 modes were found for each value of ceramic dielectric constant. The reason we swept over dielectric constant is that although the nominal value is 10 , this value is notoriously variable for ceramic and has been seen to vary by up to $50 \%$ in earlier studies.

We used the Poisson 2 code to generate the necessary gun solution for gun voltages between $10 \mathrm{KV}$ and $550 \mathrm{KV}$ in $10 \mathrm{KV}$ increments. After the modes and gun solutions were obtained, the perturbation method described herein was used to generate the stability ratio $(\mathrm{Qm} / \mathrm{Qb})$ and power balance for all modes at all gun voltages. The results of this computation are shown in Figs. 5-7.

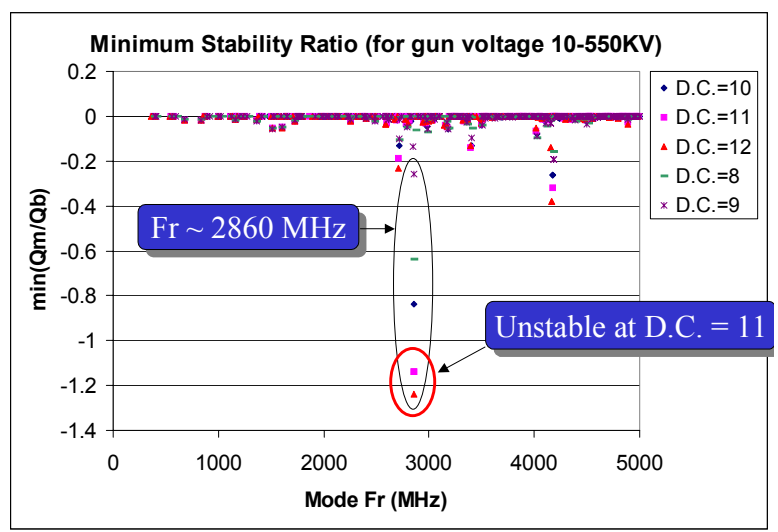

Figure 5: Minimum values of stability ratio $(<-1$ indicate instability) for all monopole modes resonating at $<5 \mathrm{GHz}$ in klystron gun region ( $\sim 215$ modes) for a ceramic window dielectric constant varying from $8-12$. Reported minimum for each mode is over range of gun voltages $10 \mathrm{KV}-550 \mathrm{KV}$.

Of the modes resonant below $5 \mathrm{GHz}$, only one at 2860 $\mathrm{MHz}$ shows potential instability as seen in Fig. 5. This mode is predicted to be stable at the nominal ceramic window dielectric constant of 10 , but is unstable if the dielectric constant is 11 .

The stability ratio for the unstable mode is plotted as a function of gun voltage in Fig. 6, showing that the onset of instability occurs for a gun voltage of $440 \mathrm{KV}$. This mode can therefore be induced during the ramp-up in beam voltage, potentially disrupting tube start. The field pattern for the unstable mode is shown in Fig. 7.

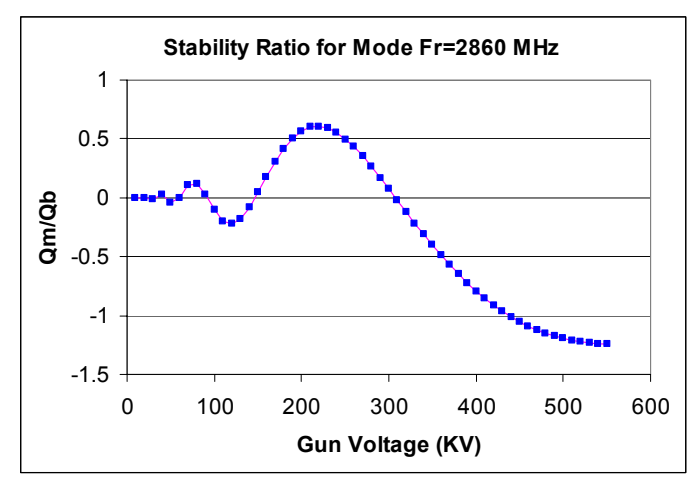

Figure 6: Stability ratio values for $2860 \mathrm{MHz}$ mode in gun region for $\mathrm{A}-\mathrm{K}$ voltages in the range $10 \mathrm{KV}-550 \mathrm{KV}$ for window dielectric constant of 12 . Mode is unstable if this ratio is $<-1$, so this mode will grow for gun voltages $>$ $440 \mathrm{KV}$

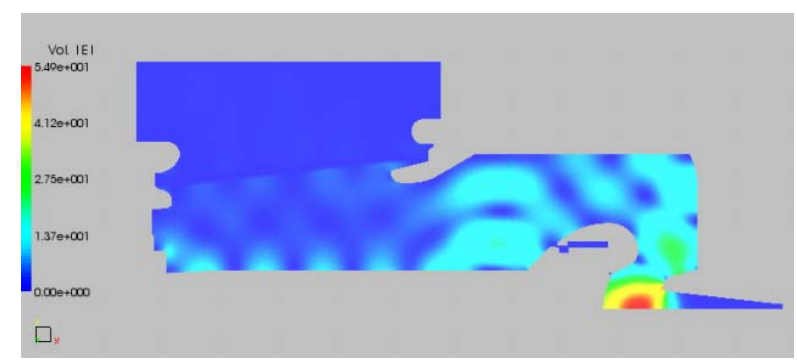

Figure 7: $|\mathrm{E}|$ of potentially unstable mode.

\section{CONCLUSIONS}

A method for computing beam-loaded Q values based on an electrostatic PIC calculation is presented. This procedure was validated against established results, and then used to analyze the gun region of the SLAC XP-4 high-power klystron. The method predicts an unstable mode at $2860 \mathrm{MHz}$ for certain values of the dielectric constant of the ceramic RF window in this structure.

\section{REFERENCES}

[1] B. Krietenstein, et al., "Spurious oscillations in highpower klystrons," PAC'95, 1995.

[2] U. Becker, et al., "Simulation of oscillations in highpower klystrons," Proceedings of EPAC'96, 1996, p. 1274-1276.

[3] V. Ivanov, "The mathematical methods and electromagnetic codes for the physical electronic devices," Proc. 2nd Int. Conf. on Computations in Electromagnetism, Nottingham, May 13-15, 1994.

[4] G. M. Branch, "Electron beam coupling in interaction gaps of cylindrical symmetry," 1961, IRE Transactions on Electron Devices, May 1961.

[5] D. Sprehn, personal communication. 